\title{
Better Predicate Testing
}

\author{
Gary Kaminski \\ Software Engineering \\ George Mason University \\ Fairfax VA, USA \\ gkaminsk@gmu.edu
}

\author{
Paul Ammann \\ Software Engineering \\ George Mason University \\ Fairfax VA, USA \\ pammann@gmu.edu
}

\author{
Jeff Offutt \\ Software Engineering \\ George Mason University \\ Fairfax VA, USA \\ offutt@gmu.edu
}

\begin{abstract}
Mutation testing is widely recognized as being extremely powerful, but is considered difficult to automate enough for practical use. This paper theoretically addresses two possible reasons for this: the generation of redundant mutants and the lack of integration of mutation analysis with other test criteria. By addressing these two issues, this paper brings an important mutation operator, relational-operatorreplacement (ROR), closer to practical use. First, we develop fault hierarchies for the six relational operators, each of which generates seven mutants per clause. These hierarchies show that, for any given clause, only three mutants are necessary. This theoretical result can be integrated easily into mutation analysis tools, thereby eliminating generation of $57 \%$ of the ROR mutants. Second, we show how to bring the power of the ROR operator to the widely used Multiple Condition-Decision Coverage (MCDC) test criterion. This theoretical result includes an algorithm to transform any MCDC-adequate test set into a test set that also satisfies RORG, a new version of ROR appropriate for the MCDC context. The transformation does not use traditional mutation analysis, so can easily be integrated into existing MCDC tools and processes.
\end{abstract}

\section{Categories and Subject Descriptors}

D.2.5 [Software Engineering]: Testing and DebuggingTesting tools

\section{General Terms}

Verification

\section{Keywords}

Logic testing, Mutation

\section{INTRODUCTION}

The ability for mutation testing $[7,8]$ to help testers design high quality tests has always depended directly on the

Permission to make digital or hard copies of all or part of this work for personal or classroom use is granted without fee provided that copies are not made or distributed for profit or commercial advantage and that copies bear this notice and the full citation on the first page. To copy otherwise, to republish, to post on servers or to redistribute to lists, requires prior specific permission and/or a fee.

ICSE' 11, May 21-28, 2011, Waikiki, Honolulu, HI, USA

Copyright 2011 ACM 978-1-4503-0592-1/11/05 ...\$10.00. mutation operators. In program-based mutation testing, a mutation operator is a rule that specifies changes to syntactic elements in a program. A well designed set of operators can result in very powerful testing, but a poorly designed set can result in ineffective tests. Mutation operators have been designed for several programming languages, including COBOL [10], Fortran 77 [8, 19], C [6], Ada [25], and Java $[18,22]$. Jia and Harman recently surveyed mutation analysis for programs and other software engineering artifacts [12]. The statement-level operators have been fairly stable since the Mothra project [19], with the most important suggestion for change being from the selective operator study [23], where it was found that using five Mothra mutation operators would yield tests that killed most other mutants.

However, users of mutation have observed that mutation creates many test requirements (that is, mutants) relative to the number of tests needed when compared to other test criteria. For example, Li et al. found that mutation yielded fewer tests than the edge-pair, all-uses and prime path criteria, even though it had far more test requirements [21]. The only reasonable conclusion from this result is that the mutants somehow "overlap" in the tests needed to kill them, and probably in their ability to find faults. In other words, some mutants appear to be redundant.

The development of fault detection hierarchies, most notably the DNF fault hierarchy of Lau and Yu [20], offers a way to deal with the problem of redundant mutants. A fault hierarchy describes a dominance relation among fault classes. If a test set detects faults in a given class in the hierarchy, those tests are also guaranteed to detect faults in classes that class dominates. From the mutation analysis perspective, this means that if a fault hierarchy is built for a set of mutants, there is no need to generate mutants that are dominated by other mutants.

A closely related approach to increasing the effectiveness of each mutant is the notion of a subsuming Higher-OrderMutant (HOM) [11]. A subsuming HOM is built by combining several mutations in such a way that the combined mutant (the HOM) subsumes all of its constituent mutants. A key property of a subsuming HOM is that a test that kills the HOM is also guaranteed to kill the subsumed mutants, and hence these mutants do not need to be generated. Kaminski and Ammann put both of these ideas together in the context of mutation analysis of logic expressions in disjunctive normal form (DNF) [13]. They showed that not only are many redundant mutants generated for logic expressions, worse, these mutants miss many faults in the Lau and $\mathrm{Yu}$ fault hierarchy. They further showed how to automatically con- 
struct a small number of subsuming HOMs that guarantee detection of the entire Lau and Yu fault hierarchy-and, by subsumption, all mutants generated by a typical mutation analysis tool on the boolean structure of logic expressions.

This paper considers the relational operators that commonly appear in boolean expressions. In terms of mutation analysis, this means considering the relational operator replacement (ROR) operator. Our first contribution is to develop fault hierarchies for the mutants generated by the ROR operator. These hierarchies show that of the seven mutants generated by a single application of an ROR operator, only three are necessary.

The second contribution is to increase the strength of the logic coverage test criterion of Multiple Condition-Decision Coverage (MCDC) [5]. Logic coverage criteria such as MCDC and ACC $[5,2]$ test predicates at the clause level. That is, in a predicate $p=a \wedge b \vee c$, logic criteria test the individual clauses $(a, b, c)$. Mutation analysis, however, uses the ROR operator to test at a lower level of abstraction, inside the clauses. Thus, if $a \equiv(x>y), b \equiv(m<=n)$, and $c \equiv(d==e)$, ROR tests whether the relations inside the clauses are formulated correctly. This paper defines a stronger version of MCDC that leverages the ROR fault hierarchies to integrate the power of the ROR mutation operator into the logic criteria. This result includes an algorithm that augments an MCDC-adequate test set to also be adequate with respect to RORG, a variant of ROR appropriate for integration with MCDC.

MCDC, which is equivalent to Active Clause Coverage (ACC) $[2,1]$, is required by the US Federal Aviation Administration to test safety critical systems [26], and comes in several slightly varying versions [4]. As it turns out, any ROR-adequate test set is guaranteed also to satisfy the weakest version of MCDC [1]. However, the converse is not true; an MCDC-adequate test set does not necessarily satisfy ROR coverage. Intuitively, this is because of the difference in the previous paragraph; MCDC treats predicates as boolean functions and ignores relational operators in these expressions. For applications where faults in relational operators are a concern, including ROR can clearly lead to stronger tests.

The organization of this paper is as follows. Section 2 describes background in mutation operators, logic mutation operators, and recent results in logic testing. Section 3 explores the relationship between the ROR mutation operator and logic criteria and section 4 presents a new ROR fault hierarchy. Section 5 presents a new version of the ROR operator that is just as effective but that produces less than half the number of mutants. Section 6 presents modified versions of MCDC that are more effective and only slightly more expensive in terms of tests required. Section 7 offers conclusions and recommendations for future automation.

\section{BACKGROUND}

The classic definition of the $R O R$ operator is from the 1991 detailed description of the Mothra mutation system [19]: Each occurrence of a relational operator $(<,>, \leq, \geq$, $=, \neq)$ is replaced by each other operator and the expression is replaced by True and False. Most mutation systems (including muJava [22]) since Mothra have implemented these operators following these definitions.

The literature contains several test coverage criteria that focus on the logical structure of predicates. This literature assumes that a predicate is assembled from boolean valued clauses via the standard boolean operators, typically including not $(\neg)$, and $(\wedge)$, and or $(\vee)$. Significantly, from the perspective of this paper, the internal structure of the clauses is ignored. The most powerful logic coverage criteria is combinatorial coverage, which requires every possible assignment of truth values to clauses. In a predicate with $n$ clauses, combinatorial coverage requires $2^{n}$ tests.

Other logic coverage criteria ask for some subset of the possible $2^{n}$ tests defined by combinatorial coverage. Clause coverage requires each clause to take on the values true and false, and can be satisfied with just two tests. Predicate coverage requires the predicate as a whole to take on the values true and false, and can be also be satisfied with just two tests. Clause coverage and predicate coverage are both fairly weak, and neither subsumes the other.

Modified Condition Decision Coverage (MCDC) [5], which is equivalent to Active Clause Coverage (ACC) [1], is widely perceived as a powerful test coverage technique, and is used in the certification of safety critical systems [26]. The idea behind MCDC is that each clause should be tested to be both true and false under circumstances where the clause "matters," where this is interpreted to mean that changing the value of the clause necessarily changes the value of the predicate. Note that MCDC test requirements come in pairs-one requirement for the clause true and one for the clause false. MCDC comes in various forms depending on whether each test pair faces additional constraints beyond what is mentioned above, but the details of these forms are independent of the contributions of this paper.

A different approach to logic coverage bases it on fault detection power with respect to the Lau and $\mathrm{Yu}$ fault hierarchy [20]. The most powerful of the these techniques is MUMCUT [3], which is guaranteed to detect the entire hierarchy. MUMCUT generally only applies to predicates written in Disjunctive Normal Form (DNF), although extensions to more general forms have been explored [27]. MUMCUT generates many unnecessary tests; various refinements of MUMCUT have addressed this shortcoming [9, 14, 15, $16]$.

\section{ROR AND LOGIC CRITERIA}

This section proves that logic coverage criteria do not subsume ROR mutation testing. For convenience, we use the term ROR mutation to mean mutation using just the ROR operator. The strongest logic coverage criterion is combinatorial coverage, which requires that a predicate be tested with all combinations of truth values. Consider the twoclause predicate $a<b \vee c<d$. Combinatorial coverage requires the tests TT, TF, FT, FF. The following assignments to $a, b, c$, and $d$ satisfy combinatorial coverage:

$$
\begin{aligned}
& a=1, b=2, c=1, d=2(T T) \\
& a=1, b=2, c=2, d=1(T F) \\
& a=2, b=1, c=1, d=2(\text { FT) }
\end{aligned}
$$$$
a=2, b=1, c=2, d=1(F F)
$$

However, none of these four tests detects either of the two ROR mutants where $<$ is replaced by $<=$. To detect the mutant where $a<b$ is changed to $a<=b, a$ and $b$ must have the same value. Thus, combinatorial coverage does not subsume ROR mutation. Combinatorial coverage is the strongest logic criterion and subsumes all others, thus no logic coverage criterion can subsume ROR mutation. Intuitively, the logic coverage criteria treat each clause as a 
unit, as a boolean variable, ignoring any structure inside the clause such as the relational operators. The ROR operator, on the other hand, explicitly requires tests to evaluate whether the correct relational operator was used. That is, logic criteria test the clause level, whereas the ROR operator tests the relational level, a more detailed level of abstraction.

\section{A NEW ROR FAULT HIERARCHY}

Mutation is widely considered to be expensive, but "expense" can be measured in several ways. Li et al. [21] found that although mutation creates more test requirements (that is, mutants) than other test criteria, it does not need more tests. The obvious implication from that result is that many mutants are unnecessary or redundant. The selective operator study [23] reduced the number of mutants by an order of magnitude, but mutation systems such as muJava [22] still generate more mutants than are necessary. (Li et al.'s study used the muJava selective set of mutation operators.)

This section analyzes the ROR mutation operator on a case by case basis. For each relational operator, the conditions under which mutants created by that operator will be killed are derived. These are called detection conditions. From that, a hierarchy of mutants is formed. The table in figure 1 shows the detection conditions for each mutant of the $<$ operator. When $a<b$ is mutated to False, the detection condition is $a<b$ and the value for $a<b$ must be True. Likewise, if the mutant is $a<=b$, the detection condition is $a==b$ and the value must be False.

This leads to the mutant class hierarchy for $<$ in figure 1. The arrows imply a dominance in the sense of Lau and $\mathrm{Yu}$ [20]. If a test kills the mutant where $<$ is replaced by False, that test is guaranteed to also kill the mutants where $<$ is replaced by $==$ and $>$, and by transitivity, the mutant where $<$ is replaced by $>=$.

\begin{tabular}{|l|l|l|}
\hline \multicolumn{1}{|c|}{ Mutant } & $\begin{array}{l}\text { Detection } \\
\text { condition }\end{array}$ & $\begin{array}{c}\text { Value of } \\
a<b\end{array}$ \\
\hline$<$ replaced by $\mathrm{F}$ & $a<b$ & $\mathrm{~T}$ \\
\hline$<$ replaced by $<=$ & $a==b$ & $\mathrm{~F}$ \\
\hline$<$ replaced by $!=$ & $a>b$ & $\mathrm{~F}$ \\
\hline$<$ replaced by $==$ & $a<=b$ & $\mathrm{~T}$ or $\mathrm{F}$ \\
\hline$<$ replaced by $>$ & $a !=b$ & $\mathrm{~T}$ or $\mathrm{F}$ \\
\hline$<$ replaced by $\mathrm{T}$ & $a>=b$ & $\mathrm{~F}$ \\
\hline$<$ replaced by $>=$ & $\mathrm{T}$ & $\mathrm{T}$ or $\mathrm{F}$ \\
\hline
\end{tabular}

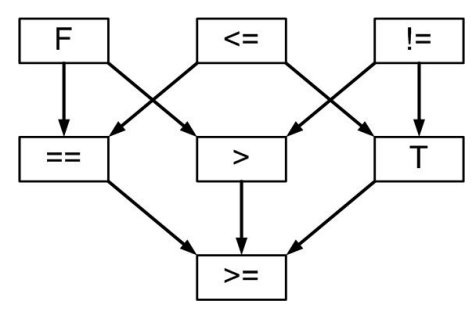

Figure 1: Mutants, Detection Conditions, and Class Hierarchy for <

Figures 2 through 6 show the detection conditions and mutant hierarchies for the other relational operators. These results hold for all programming and specification languages that use relational operators as defined in standard mathematics.

\begin{tabular}{|c|l|l|}
\hline \multicolumn{1}{|c|}{ Mutant } & $\begin{array}{l}\text { Detection } \\
\text { condition }\end{array}$ & $\begin{array}{c}\text { Value of } \\
a>b\end{array}$ \\
\hline$>$ replaced by $\mathrm{F}$ & $a>b$ & $\mathrm{~T}$ \\
\hline$>$ replaced by $>=$ & $a==b$ & $\mathrm{~F}$ \\
\hline$>$ replaced by $!=$ & $a<b$ & $\mathrm{~F}$ \\
\hline$>$ replaced by $==$ & $a>=b$ & $\mathrm{~T}$ or $\mathrm{F}$ \\
\hline$>$ replaced by $<$ & $a !=b$ & $\mathrm{~T}$ or $\mathrm{F}$ \\
\hline$>$ replaced by $\mathrm{T}$ & $a<=b$ & $\mathrm{~F}$ \\
\hline$>$ replaced by $<=$ & $\mathrm{T}$ & $\mathrm{T}$ or $\mathrm{F}$ \\
\hline
\end{tabular}

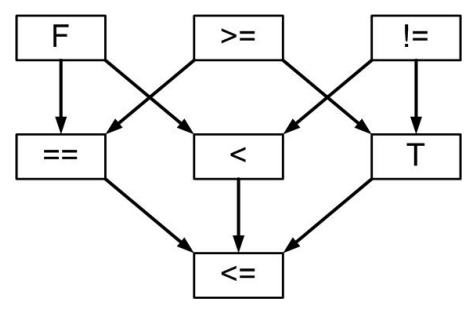

Figure 2: Mutants, Detection Conditions, and Class Hierarchy for $>$

\begin{tabular}{|l|l|l|}
\hline \multicolumn{1}{|c|}{ Mutant } & $\begin{array}{c}\text { Detection } \\
\text { condition }\end{array}$ & $\begin{array}{c}\text { Value of } \\
a<=b\end{array}$ \\
\hline$<=$ replaced by $\mathrm{T}$ & $a>b$ & $\mathrm{~F}$ \\
\hline$<=$ replaced by $<$ & $a==b$ & $\mathrm{~T}$ \\
\hline$<=$ replaced by $==$ & $a<b$ & $\mathrm{~T}$ \\
\hline$<=$ replaced by $!=$ & $a>=b$ & $\mathrm{~T}$ or $\mathrm{F}$ \\
\hline$<=$ replaced by $>=$ & $a !=b$ & $\mathrm{~T}$ or $\mathrm{F}$ \\
\hline$<=$ replaced by $\mathrm{F}$ & $a<=b$ & $\mathrm{~T}$ \\
\hline$<=$ replaced by $>$ & $\mathrm{T}$ & $\mathrm{T}$ or $\mathrm{F}$ \\
\hline
\end{tabular}

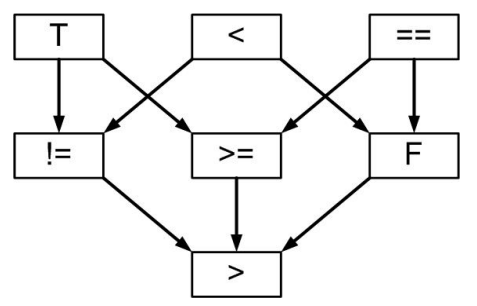

Figure 3: Mutants, Detection Conditions, and Class Hierarchy for $<=$

For all six relational operators, we can immediately see that tests that detect three of the ROR mutants are guaranteed to detect all seven ROR mutants. Conversely, if there is no arrow in a hierarchy from one mutant to another, a test that detects the first mutant is guaranteed not to detect the other. For example, consider figure 1. A test that detects the mutant where $<$ is replaced with $<=$ will never detect the mutant where $<$ is replaced with $>$. Also any test that detects a mutant at the top level of a hierarchy is guaranteed not to detect either of the other two mutants at the top level of the hierarchy. For example, again consider figure 1. A test that detects the mutant where $<$ is replaced with ! = will never detect the mutant where $<$ is replaced with False.

Detecting all ROR mutants guarantees clause coverage. Both $\mathrm{T}$ and $\mathrm{F}$ appear at least once among the top three rows in the "Value of a relop b" column in each table, which achieves clause coverage. 


\begin{tabular}{|c|l|l|}
\hline \multicolumn{1}{|c|}{ Mutant } & $\begin{array}{l}\text { Detection } \\
\text { condition }\end{array}$ & $\begin{array}{c}\text { Value of } \\
a>=b\end{array}$ \\
\hline$>=$ replaced by $\mathrm{T}$ & $a<b$ & $\mathrm{~F}$ \\
\hline$>=$ replaced by $>$ & $a==b$ & $\mathrm{~T}$ \\
\hline$>=$ replaced by $==$ & $a>b$ & $\mathrm{~T}$ \\
\hline$>=$ replaced by $!=$ & $a<=b$ & T or $\mathrm{F}$ \\
\hline$>=$ replaced by $<=$ & $a !=b$ & T or $\mathrm{F}$ \\
\hline$>=$ replaced by $\mathrm{F}$ & $a>=b$ & $\mathrm{~T}$ \\
\hline$>=$ replaced by $<$ & $\mathrm{T}$ & $\mathrm{T}$ or $\mathrm{F}$ \\
\hline
\end{tabular}

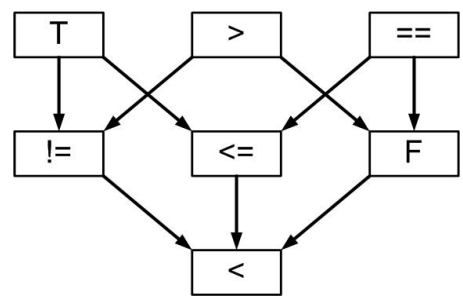

Figure 4: Mutants, Detection Conditions, and Class Hierarchy for $>=$

\begin{tabular}{|l|l|l|}
\hline \multicolumn{1}{|c|}{ Mutant } & $\begin{array}{l}\text { Detection } \\
\text { condition }\end{array}$ & $\begin{array}{c}\text { Value of } \\
a==b\end{array}$ \\
\hline$==$ replaced by $\mathrm{F}$ & $a==b$ & $\mathrm{~T}$ \\
\hline$==$ replaced by $<=$ & $a<b$ & $\mathrm{~F}$ \\
\hline$==$ replaced by $>=$ & $a>b$ & $\mathrm{~F}$ \\
\hline$==$ replaced by $<$ & $a<=b$ & $\mathrm{~T}$ or $\mathrm{F}$ \\
\hline$==$ replaced by $>$ & $a>=b$ & $\mathrm{~T}$ or $\mathrm{F}$ \\
\hline$==$ replaced by $\mathrm{T}$ & $a !=b$ & $\mathrm{~F}$ \\
\hline$==$ replaced by $!=$ & $\mathrm{T}$ & $\mathrm{T}$ or $\mathrm{F}$ \\
\hline
\end{tabular}

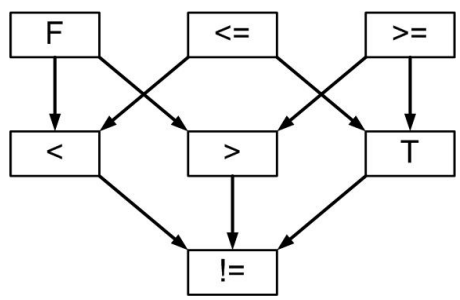

Figure 5: Mutants, Detection Conditions, and Class Hierarchy for $==$

\section{A CHEAPER ROR OPERATOR}

The detection conditions and mutant hierarchies in section 4 lead to an immediate result. The classic definition of the ROR operator [19] should be reformulated to only create the three mutants on top of the mutant hierarchy for the relational operator being mutated. Specifically, if the operator is $<$, only $<=, !=$, and False should be created; if the operator is $>$, only $>=, !=$, and False should be created; if the operator is $<=$, only $<,==$, and True should be created; if the operator is $>=$, only $>,==$, and True should be created; if the operator is $==$, only $<=,>=$, and False should be created; and if the operator is $!=$, only $<,>$, and True should be created. This is an immediate savings of four mutants for each relational operator.

\section{STRONGER LOGIC CRITERIA}

As described in section 3, logic coverage criteria such as MCDC, ACC, and MUMCUT [3] (an extremely powerful

\begin{tabular}{|l|l|l|}
\hline \multicolumn{1}{|c|}{ Mutant } & $\begin{array}{c}\text { Detection } \\
\text { condition }\end{array}$ & $\begin{array}{c}\text { Value of } \\
a !=b\end{array}$ \\
\hline$!=$ replaced by $\mathrm{T}$ & $a==b$ & $\mathrm{~F}$ \\
\hline$!=$ replaced by $<$ & $a>b$ & $\mathrm{~T}$ \\
\hline$!=$ replaced by $>$ & $a<b$ & $\mathrm{~T}$ \\
\hline$!=$ replaced by $<=$ & $a>=b$ & $\mathrm{~T}$ or $\mathrm{F}$ \\
\hline$!=$ replaced by $>=$ & $a<=b$ & $\mathrm{~T}$ or $\mathrm{F}$ \\
\hline$!=$ replaced by $\mathrm{F}$ & $a !=b$ & $\mathrm{~T}$ \\
\hline$!=$ replaced by $==$ & $\mathrm{T}$ & $\mathrm{T}$ or $\mathrm{F}$ \\
\hline
\end{tabular}

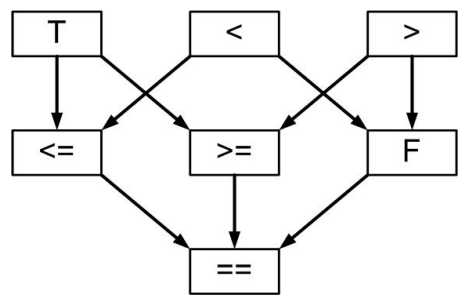

Figure 6: Mutants, Detection Conditions, and Class Hierarchy for ! =

coverage criterion for boolean expressions in Disjunctive Normal Form) do not test inside the clauses at the relational operator level. This section introduces a modification of the MCDC criterion to include the additional tests required by the ROR mutation operator to test relational expressions. The general approach works for all logic criteria; we illustrate it with MCDC because of its wide use in the aerospace industry through FAA requirements [26].

MCDC coverage of a predicate with $N$ clauses requires at least $N+1$ and no more than $2 N$ tests. Making an MCDC test set ROR-adequate adds at most $N$ additional tests.

Mutation operators and logic coverage criteria have a theoretical difference that affects this construction. MCDC is considered to be a semantic coverage criterion [17], whereas mutation is considered to be syntactic. Semantic criteria generate test requirements independently of how the predicates are written, whereas syntactic criteria do not. For example, if the same clause appears twice in a predicate $(p=a \vee(b \wedge a))$, semantic criteria like MCDC and ACC are not affected; when a value for $a$ is chosen, the same value is used for all occurrences of $a$. Mutation, on the other hand, will mutate each occurrence of $a$ independently. If $a=a 1>a 2$, this means we would have 14 mutants rather than 7 , and possibly need to have the first occurrence of $a$ have one value, and the second occurrence a different value to kill a mutant.

To successfully integrate a syntactic criterion like ROR mutation with the semantic criterion of MCDC, we need to make certain adaptions. Our approach is to use the concept of a higher-order-mutation operator (HOM) [11], which is a mutation operator that defines more than one change. Our HOM, the Relational Operator Replacement Global (RORG), is defined as follows. Consider a boolean expression $p$ with $n$ boolean clauses $c_{1}, c_{2}, \ldots, c_{n}$. Each boolean clause $c_{i}$ appears at least once and may appear more than once in $p$. For each boolean clause $c_{i}$ that uses a relational operator, $c_{i}=a_{1}$ relop $a_{2}$, RORG replaces $e v$ ery occurrence of $c_{i}$ with one of the ROR mutations. This means RORG is essentially a semantic, rather than a syntactic, mutation operator. For example, given the predicate $p=a 1>a 2 \vee(b 1==b 2 \wedge a 1>a 2)$, two RORG mu- 
tants are: $p=a 1>=a 2 \vee(b 1==b 2 \wedge a 1>=a 2)$ and $p=a 1 !=a 2 \vee(b 1==b 2 \wedge a 1 !=a 2)$.

With the RORG adaptation of ROR, we can develop an algorithm that adds RORG-adequacy to an MCDC test set. The idea behind the algorithm is quite simple. We identify tests that satisfy MCDC with respect to each boolean clause $c$. The MCDC requirements on these tests are that $c$ take on the values True and False under conditions where $c$ determines the value of predicate $p$. We then note that the top three rows of every table in section 4 have the same three detection conditions, $<,==$, and $>$. MCDC by itself requires two tests for each clause (True and False). So at least two of these will have been satisfied by a test where $c$ determines the value of $p$. First, the algorithm identifies whether one of the three detection conditions is not satisfied by the MCDC tests, and which. Then it adds a test to satisfy the third. This process is shown in pseudo-code form in algorithm 1 .

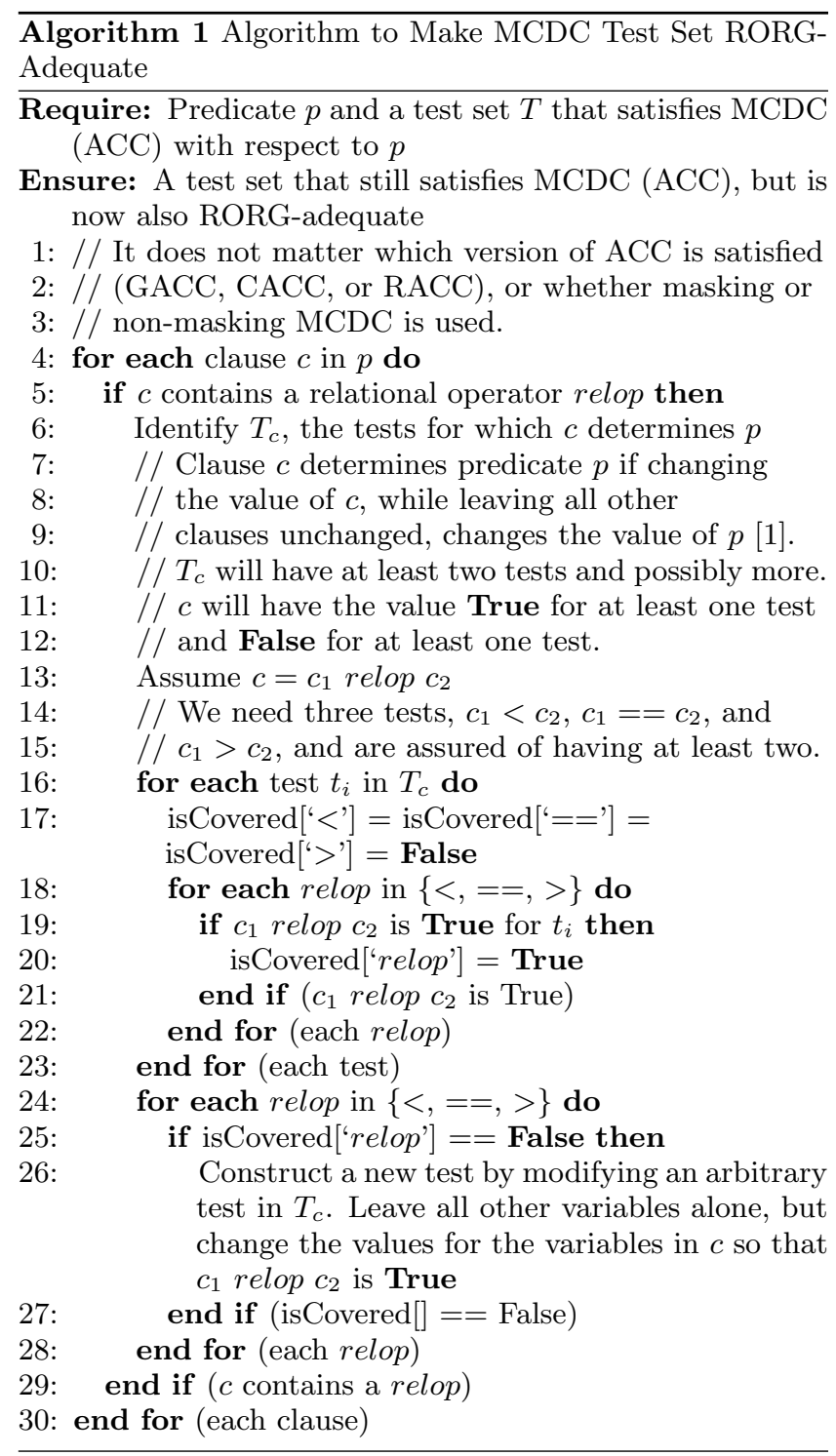

Algorithm Proof Sketch: The algorithm makes two claims about the resulting test set. The first is that it satisfies MCDC. Since the input test set satisfies MCDC, and no tests are removed from this set, it is clear that the output still satisfies MCDC.

The second claim is that the output test set kills every RORG mutant. We prove this via weak mutation analysis, which is arguably the only generally applicable approach in the context of MCDC. Weak mutation analysis requires infection of the subsequent state to kill a mutant. Offutt and Lee [24] found that infection in the context of a mutated predicate is best defined as the final value of the predicate being incorrect. Thus, for each RORG mutant there must be at least one test that causes the predicate to evaluate to the wrong value. Inspection of the detection conditions in figures 1 through 6 shows that to kill all ROR mutants, the relation between $c_{1}$ and $c_{2}$ in $c=c_{1}$ relop $c_{2}$ must have all the three possible values, namely: $c_{1}<c_{2}, c_{1}==c_{2}$, and $c_{1}>c_{2}$. For these detection conditions to cause predicate $p$ to evaluate to a different result, it is necessary that $c$ determines $p$. Tests in $T_{c}$ satisfy the constraint that $c$ determines $p$ by construction. In addition, any new test (possibly) generated by the algorithm for clause $c$ necessarily also satisfies the constraint that $c$ determines $p$. The algorithm forces the three possible relations between $c_{1}$ and $c_{2}$ for tests where $c$ determines $p$. Hence RORG is satisfied.

Note that the resulting test set does not necessarily satisfy ROR. The reason is that if we consider each clause $c$ syntactically as it appears in the predicate, there is no guarantee that the original MCDC test set has any tests in the set $T_{c}$. However, for RORG, each $T_{c}$ is guaranteed to always be at least of size 2, and, further, to satisfy predicate coverage. Complexity is on the order of the product of the number of tests and the number of clauses. If the test set is specifically optimized for MCDC, the size of the test set is linear in the number of clauses. Hence, in this case, the algorithm is quadratic in the number of clauses.

Example: Consider the predicate $p=a \wedge b \vee c$, where $a=\left(a_{1}<a_{2}\right), b=\left(b_{1} \leq b_{2}\right)$, and $c=\left(c_{1}==c_{2}\right)$.

The following test set satisfies the most restrictive MCDC coverage criterion (RACC): $T=\left\{t_{1}, t_{2}, t_{3}, t_{4}\right\}=$ $\{T T F, T F T, T F F, F T F\}$. These tests are refined to have the value assignments:

$t_{1}: a_{1}=5, a_{2}=6, b_{1}=10, b_{2}=11, c_{1}=21, c_{2}=22$

$t_{2}: a_{1}=5, a_{2}=6, b_{1}=11, b_{2}=10, c_{1}=21, c_{2}=21$

$t_{3}: a_{1}=5, a_{2}=6, b_{1}=11, b_{2}=10, c_{1}=21, c_{2}=22$

$t_{4}: a_{1}=6, a_{2}=5, b_{1}=10, b_{2}=11, c_{1}=21, c_{2}=22$

We first consider clause $a$ from line 4 in algorithm 1 . The set $T_{a}$ of tests where $a$ determines $p$ is $\left\{t_{1}, t_{4}\right\}$. Test $t_{1}$ satisfies $a_{1}<a_{2}$ and $t_{4}$ satisfies $a_{1}>a_{2}$, so the algorithm adds a new test (in line 26) to satisfy $a_{1}==a_{2}$. Any test for which $a$ determines $p$ will do, so the algorithm starts with $t_{1}$, and modifies the values for $a_{1}$ and $a_{2}$ to be $a_{1}=5$ and $a_{2}=5$.

For clause $b$, the set $T_{b}$ of tests where $b$ determines $p$ is $\left\{t_{1}, t_{3}\right\}$. Test $t_{1}$ satisfies $b_{1}<b_{2}$ and $t_{3}$ satisfies $b_{1}>b_{2}$, so the algorithm adds a new test (in line 26) to satisfy $b_{1}$ $==b_{2}$. Any test for which $b$ determines $p$ will do, so the algorithm starts with $t_{1}$, and modifies the values for $b_{1}$ and $b_{2}$ to be $b_{1}=10$ and $b_{2}=10$.

Finally, for clause $c$, the set $T_{c}$ of tests where $c$ determines $p$ is $\left\{t_{2}, t_{3}\right\}$. Test $t_{2}$ satisfies $c_{1}==c_{2}$ and $t_{3}$ satisfies $c_{1}<$ $c_{2}$, so the algorithm adds a new test (in line 26) to satisfy $c_{1}>c_{2}$. Any test for which $c$ determines $p$ will do, so the algorithm starts with $t_{2}$, and modifies the values for $c_{1}$ and $c_{2}$ to be $c_{1}=22$ and $c_{2}=21$. 
The resulting MCDC/RORG-adequate test set is shown in table 1. The last three tests marked "New" are added by the algorithm. The original tests they were derived from are included in parentheses, and the modified values are shown in bold. The additional detection conditions that were covered are shown in the last three columns.

Table 1: Example of expanding MCDC tests to be RORG adequate

\begin{tabular}{|c|c|c|c|c|c|c|c|c|c|c|}
\hline Test & Value & $a_{1}$ & $a_{2}$ & $b_{1}$ & $b_{2}$ & $c_{1}$ & $c_{2}$ & $a$ & $b$ & $c$ \\
\hline$t_{1}$ & TTF & 5 & 6 & 10 & 11 & 21 & 22 & $<$ & $<$ & \\
\hline$t_{2}$ & TFT & 5 & 6 & 11 & 10 & 21 & 21 & & & $==$ \\
\hline$t_{3}$ & TFF & 5 & 6 & 10 & 11 & 21 & 22 & & $>$ & $<$ \\
\hline$t_{4}$ & FTF & 6 & 5 & 10 & 11 & 21 & 22 & $>$ & & \\
\hline New & $\left(t_{1}\right)$ & 5 & 5 & 10 & 11 & 21 & 22 & $==$ & & \\
\hline New & $\left(t_{1}\right)$ & 5 & 6 & 10 & 10 & 21 & 22 & & $==$ & \\
\hline New & $\left(t_{2}\right)$ & 5 & 6 & 11 & 10 & 22 & 21 & & & $>$ \\
\hline
\end{tabular}

\section{CONCLUSIONS AND RECOMMENDA- TIONS}

This paper presents two theoretical results, a way to reduce the number of mutants generated for the ROR operator by eliminating redundancy among mutants, and a way to strengthen logic criteria such as MCDC by using the ROR mutation operator to increase precision.

The first result can be used to improve future mutation tools by reducing the number of mutants generated. The traditional ROR operator creates seven mutants for each relational operator, of which four are provably redundant. We believe it is likely that existing mutation systems produce lots of similarly redundant mutants, and similar analysis could greatly reduce the number of mutants created by future mutation systems. In mutation testing, each mutant represents a test requirement, so reducing the number of mutants created can have a major impact on the automation of mutation testing.

The second result can be used to strengthen logic test criteria. Logic testing criteria have traditionally only looked at the clause level, treating each clause as a simple boolean variable. Part of the power of mutation stems from the fact that it looks inside clauses, and tries to determine whether a clause such as $a>b$ is correctly formulated. By adding one more test for each clause, and using the redundancy proofs for the ROR operator, this paper shows how logic criteria can be extended to gain this power. The algorithm in section 6 shows how this technique can easily be incorporated into an automated test tool.

This result is particularly significant for the MCDC criterion [5], because it is mandated for certain safety-critical software components on aircrafts and air traffic controllers by the US Federal Aviation Administration [26]. The simple extension to MCDC presented in this paper has the ability to strengthen testing of this crucial software, potentially making air travel safer. Although the algorithm is definitive in terms of adding RORG-adequacy to an MCDC-adequate test set, empirical studies are needed to determine how much augmenting MCDC test sets with RORG-adequacy improves fault detection. The algorithm can also be used in the automation of testing virtually any kind of control software, much of which makes heavy use of logical predicates and much of which is safety critical.

\section{REFERENCES}

[1] P. Ammann and J. Offutt. Introduction to Software Testing. Cambridge University Press, Cambridge, UK, 2008. ISBN 0-52188-038-1.

[2] P. Ammann, J. Offutt, and H. Huang. Coverage criteria for logical expressions. In Proceedings of the 14 th International Symposium on Software Reliability Engineering, pages 99-107, Denver, CO, November 2003. IEEE Computer Society Press.

[3] T. Y. Chen, M. F. Lau, and Y. T. Yu. MUMCUT: A fault-based strategy for testing boolean specifications. In APSEC '99: Proceedings of the Sixth Asia Pacific Software Engineering Conference, pages 606-613, Takamatsu, Japan, 1999. IEEE Computer Society Press.

[4] J. Chilenski and L. A. Richey. Definition for a masking form of modified condition decision coverage (MCDC). Technical report, Boeing, Seattle, WA, 1997. http://www.boeing.com/nosearch/mcdc/.

[5] J. J. Chilenski and S. P. Miller. Applicability of modified condition/decision coverage to software testing. Software Engineering Journal, 9(5):193-200, September 1994.

[6] M. E. Delamaro and J. C. Maldonado. Proteum-A tool for the assessment of test adequacy for $\mathrm{C}$ programs. In Proceedings of the Conference on Performability in Computing Systems (PCS 96), pages 79-95, New Brunswick, NJ, July 1996.

[7] R. A. DeMillo, R. J. Lipton, and F. G. Sayward. Hints on test data selection: Help for the practicing programmer. IEEE Computer, 11(4):34-41, April 1978.

[8] R. A. DeMillo and J. Offutt. Constraint-based automatic test data generation. IEEE Transactions on Software Engineering, 17(9):900-910, September 1991.

[9] A. Gargantini and G. Fraser. Generating minimal fault detecting test suites for boolean expressions. In AMOST 2010 - 6th Workshop on Advances in Model Based Testing, pages 37-45, Paris, France, April 2010.

[10] J. M. Hanks. Testing COBOL programs by mutation: Volume I-introduction to the CMS.1 system, volume II - CMS.1 system documentation. Technical report GIT-ICS-80/04, Georgia Institute of Technology, February 1980.

[11] Y. Jia and M. Harman. Constructing subtle faults using higher order mutation testing. In 2008 Eighth IEEE International Working Conference on Source Code Analysis and Manipulation, pages 249-258, Beijing, September 2008.

[12] Y. Jia and M. Harman. An analysis and survey of the development of mutation testing. IEEE Transactions of Software Engineering, To appear, 2010. DOI: http://doi.ieeecomputersociety.org/10.1109/TSE.2010.62.

[13] G. Kaminski and P. Ammann. Using a fault hierarchy to improve the efficiency of DNF logic mutation testing. In 2nd IEEE International Conference on Software Testing, Verification and Validation (ICST 2009), pages 386-395, Denver, CO, April 2009.

[14] G. Kaminski and P. Ammann. Using logic criterion feasibility to reduce test set size while guaranteeing fault detection. In 2nd IEEE International Conference on Software Testing, Verification and Validation 
(ICST 2009), pages 356-365, Denver, CO, April 2009.

[15] G. Kaminski and P. Ammann. Applications of optimization to logic testing. In CSTVA 2010 - 2nd Workshop on Constraints in Software Testing, Verification and Analysis, pages 331-336, Paris, France, April 2010.

[16] G. Kaminski and P. Ammann. Reducing logic test set size while preserving fault detection. Journal of Software Testing, Verification and Reliability, Wiley, to appear. Special issue from the 2009 International Conference on Software Testing, Verification and Validation.

[17] G. Kaminski, G. Williams, and P. Ammann. Reconciling perspectives of logic testing for software. Journal of Software Testing, Verification and Reliability, Wiley, 18(3):149-188, September 2008.

[18] S. Kim, J. A. Clark, and J. A. McDermid. Investigating the effectiveness of object-oriented strategies with the mutation method. In Proceedings of Mutation 2000: Mutation Testing in the Twentieth and the Twenty First Centuries, pages 4-100, San Jose, CA, October 2000. Wiley's Software Testing, Verification, and Reliability, December 2001.

[19] K. N. King and J. Offutt. A Fortran language system for mutation-based software testing. Software-Practice and Experience, 21(7):685-718, July 1991.

[20] M. F. Lau and Y. T. Yu. An extended fault class hierarchy for specification-based testing. ACM Transactions on Software Engineering Methodology, 14(3):247-276, July 2005.
[21] N. Li, U. Praphamontripong, and J. Offutt. An experimental comparison of four unit test criteria: Mutation, edge-pair, all-uses and prime path coverage. In Fifth Workshop on Mutation Analysis (Mutation 2009), Denver CO, April 2009.

[22] Y.-S. Ma, J. Offutt, and Y.-R. Kwon. MuJava : An automated class mutation system. Software Testing, Verification, and Reliability, Wiley, 15(2):97-133, June 2005.

[23] J. Offutt, A. Lee, G. Rothermel, R. Untch, and C. Zapf. An experimental determination of sufficient mutation operators. ACM Transactions on Software Engineering Methodology, 5(2):99-118, April 1996.

[24] J. Offutt and S. D. Lee. An empirical evaluation of weak mutation. IEEE Transactions on Software Engineering, 20(5):337-344, May 1994.

[25] J. Offutt, J. Payne, and J. M. Voas. Mutation operators for Ada. Technical report ISSE-TR-96-09, Department of Information and Software Engineering, George Mason University, Fairfax VA, March 1996. http://www.cs.gmu.edu/ tr_admin/.

[26] RTCA-DO-178B. Software considerations in airborne systems and equipment certification, December 1992.

[27] C. Sun, Y. Dong, R. Lai, K. Y. Sim, and T. Y. Chen. Analyzing and extending MUMCUT for fault-based testing of general boolean expressions. In The Sixth IEEE International Conference on Computer and Information Technology, pages 184-189, Seoul, Korea, September 2006. 